\title{
Clinico-Epidemiological Features and Survival Outcome in Patients with NSCLC: Ain Shams Clinical Oncology Department 5-Year Data
}

\author{
Ahmed Nagy*, Mohamed Kelney \\ Department of Clinical Oncology and Nuclear Medicine, Ain Shams University Faculty of Medicine, Cairo, Egypt \\ Email address: \\ ahmedalynagy@yahoo.com (A. Nagy), kelaney.m $@$ med.asu.edu.eg (M. Kelney) \\ ${ }^{*}$ Corresponding author \\ To cite this article: \\ Ahmed Nagy, Mohamed Kelney. Clinico-Epidemiological Features and Survival Outcome in Patients with NSCLC: Ain Shams Clinical \\ Oncology Department 5-Year Data. Cancer Research Journal. Vol. 7, No. 2, 2019, pp. 53-57. doi: 10.11648/j.crj.20190702.14
}

Received: April 28, 2019; Accepted: June 10, 2019; Published: June 24, 2019

\begin{abstract}
Background: Primary lung cancer is the most common malignant neoplasm worldwide with various prognostic factors. Methods: Data was analysed retrospectively from the medical records of 504 patients diagnosed with NSCLC and treated at Department of Clinical Oncology and Nuclear Medicine, Ain Shams University, Cairo-Egypt in the period from 1-1-2008 till 31-12- 2012. Results: The Median PFS after first, second and third lines was 3, 4 and 2 months respectively and the median OS was 8 months. Factors which were associated with a statistically significant difference in median OS were: age $<60$ years versus $\geq 60$ years ( 10 and 7 months respectively, $p<0.001$ ), female versus male gender $(10$ and 8 months respectively, $p<0.001$ ), urban versus rural residence ( 9 and 8 months respectively, $p=0.03$ ), smokers versus non-smokers $(8$ and 10 months respectively, $p<0.001$ ), patients presenting with non-neurological symptoms and those presenting with neurological symptoms ( 9 and 6 months respectively, $p<0.001)$ and the receiving treatment versus no treatment $(10$ and 5 months respectively, $p<0.001)$. Conclusion: This study shows that the active treatment of patients with NSCLC continues to have an important impact on survival. The fact that rural residence could be associated with worse OS warrants further investigation.
\end{abstract}

Keywords: NSCLC, Adenocarcinoma, Lung, Cancer

\section{Introduction}

\subsection{Background}

Lung cancer is the most common malignant neoplasm and the leading cause of cancer mortality around the world. Globally, bronchogenic carcinoma accounts for $1,350,000$ new cases and $12.4 \%$ of total new cancer cases.

Although, some improvement in survival has occured during the past few decades, malignant primary lung tumor continues to be a fatal disease. There has been a large relative rise in the incidence of lung cancer mainly non-small cell lung cancer (NSCLC) in developing countries in comparison to developed countries. Approximately half (49.9\%) of the new cases now are recorded in developing countries versus $31 \%$ back in 1980 . The top recorded 5-year patient survival rates $(14 \%)$ was observed in the United States, while in developing countries, it is around $8 \%$ [1].

Non-small cell lung cancer (NSCLC), with two major histological sub-types (namely: adenocarcinoma and squamous-cell carcinoma), representsabbout $85 \%$ of primary malignant lung neoplasms and a majority present with advanced stage at time of diagnosis. [2].

Before the era of targeted therapy and immunotherapy, conventional chemotherapy was the major component of the standard care along with palliative radiation therapy and supportive care for patients suffering from advanced and metastatic NSCLC. The standard treatment protocols are platinum-based doublets [3].

Other active drugsin platinum-based protocols include microtubule-targeted agents (paclitaxel, docetaxel, or vinorelbine) and DNA-targeted agents (gemcitabine or irinotecan). The efficacy of these combinations has been shown to be similar by a number of clinical studieswith 
response rates about 30\%-40\% [4, 5].

\subsection{Factors Affecting Survival}

The prognosis of patients with NSCLC remains poor due to a number of prognostic factors. About two thirds of the patients presentin an advanced stage. Most large phase III clinical studies have shown a median overall survival (OS) from 8 -10 months [6].

Albain et al. showed that a good ECOG performance status (PS), female gender, age $\leq 70$ years, hemoglobin level $>$ $11 \mathrm{~g} / \mathrm{dL}$, and normal lactate dehydrogenase (LDH) levels were associated with improved survival outcomes in patients with advanced NSCLC [7].

Current smoking status during therapy for lung cancer has been shown to have a negative impact on survival [8]. Pretreatment weight loss is generally regarded as a negative prognostic factor, but not all clinical studies have reliably confirmed this [9-11].

Forced expiratory volume (FEV1) more than 2L was found to be associated with better overall survival in patients with locally advanced NSCLC who undergo curative concurrent chemoradiation therapy $[9,12]$.

\section{Methods}

\subsection{Methods}

This study included the examination and analysis of data collected retrospectively from the medical records of 504 patients diagnosed with NSCLC who were treated at Department of Clinical Oncology and Nuclear Medicine, Ain Shams University, Cairo-Egypt in the period from January 2008 till December 2012. Progression free survival (PFS) was calculated as the time interval from receiving a chemotherapy protocol till disease progression (using RECIST Criteria 1.1) or death due to any cause. Overall survival (OS) was calculated as the time interval from diagnosis till death due to any cause.

\subsection{Statistical Analysis}

Frequencies and percentages were used to summarize categorical data. For continuous data, median or mean and interquartile range or standard deviation was used to describe centrality and dispersion respectively according to normality of the data.

Overall survival (OS) and Progression free survival (PFS) were evaluated with the Kaplan-Meier method and groups will be compared with the log-rank test. All variables that showed statistical significance in univariate analyses will be chosen for multivariate analyses. Cox regression will be used to calculate unadjusted and adjusted hazard ratios, 95\% confidence intervals and associated $\mathrm{p}$ values. Two sided $\mathrm{p}$ value $<0.05$ is considered as a cut-off value for statistical significance. Statistical analysis will be performed using IBM SPSS (SPSS Inc, Chicago, IL, USA).

\section{Results}

The medical records of 504 patients were analysed. The median age of the studied sample was 59 years with male predominance $(74.4 \%)$ and $59.1 \%$ were of urban residence. The most common symptom at presentation was dyspnea (49.2\%). Most patients were stage IV at presentation (73.2\%) and adenocarcinoma was the most common histology (52.6\%). About $85 \%$ of the patients received active treatment. The most common first line of treatment received was the gemcitabine/cisplatin protocol $(40.5 \%$ of the patients who received $1^{\text {st }}$ line treatment).

About $63 \%$ and $93 \%$ of the patients did not receive active treatment as $2^{\text {nd }}$ and $3^{\text {rd }}$ lines due to the deterioration of the ECOG performance status. EGFR mutation status testing was not available in Egypt at the time these patients received treatment and therefore non of the patients were offered mutation directed tyrosine kinase inhibitors (TKIs) (see table 1).

Table 1. Clinico-pathological and Epidemiological Features of the studied population.

\begin{tabular}{|c|c|c|}
\hline Factor & Frequency & Percent \\
\hline \multicolumn{3}{|l|}{ Age groups } \\
\hline $30-39$ & 18 & 3.6 \\
\hline $40-49$ & 81 & 16.1 \\
\hline $50-59$ & 156 & 31 \\
\hline $60-69$ & 185 & 36.7 \\
\hline $70-79$ & 62 & 12.3 \\
\hline $80-89$ & 2 & 0.4 \\
\hline \multicolumn{3}{|l|}{ Gender } \\
\hline Female $(F)$ & 129 & 25.6 \\
\hline Male (M) & 375 & 74.4 \\
\hline \multicolumn{3}{|l|}{ Occupation } \\
\hline Labour work (L) & 236 & 46.8 \\
\hline Not working $(\mathrm{N})$ & 125 & 24.8 \\
\hline Office work (O) & 143 & 28.4 \\
\hline \multicolumn{3}{|l|}{ Smoking } \\
\hline Heavy Smoker(HS) & 122 & 24.2 \\
\hline Non-Smoker (NS) & 163 & 32.3 \\
\hline Smoker (S) & 219 & 43.5 \\
\hline \multicolumn{3}{|l|}{ FamilyHistory } \\
\hline $\mathrm{N}$ & 443 & 87.9 \\
\hline $\mathrm{Y}$ & 61 & 12.1 \\
\hline \multicolumn{3}{|l|}{ Complaint } \\
\hline Cough (C) & 133 & 26.4 \\
\hline Dyspnea (D) & 248 & 49.2 \\
\hline Hemoptysis (H) & 15 & 3 \\
\hline Neurological (NE) & 42 & 8.3 \\
\hline Pain $(\mathrm{P})$ & 66 & 13.1 \\
\hline \multicolumn{3}{|l|}{ Stage } \\
\hline Non-Metastatic (L) & 135 & 26.8 \\
\hline Metastatic (M) & 369 & 73.2 \\
\hline \multicolumn{3}{|l|}{ Pathology } \\
\hline Adenocarcinoma (a) & 265 & 52.6 \\
\hline Squamous cell Carcinoma (s) & 102 & 20.2 \\
\hline Undifferentiated (u) & 137 & 27.2 \\
\hline \multicolumn{3}{|l|}{ First line protocol } \\
\hline Best Supportive Care (BSC) & 73 & 14.5 \\
\hline Carboplatin (C) & 23 & 4.6 \\
\hline Etoposide/Cisplatin (EP) & 58 & 11.5 \\
\hline Gemcitabine/Cisplatin (GP) & 204 & 40.5 \\
\hline Vinorbine/Cisplatin (NP) & 24 & 4.8 \\
\hline Radiation (RTH) & 38 & 7.5 \\
\hline Palliative surgery (SC) & 27 & 5.4 \\
\hline
\end{tabular}




\begin{tabular}{lll}
\hline Factor & Frequency & Percent \\
\hline Whole brain Radiation (WBI) & 57 & 11.3 \\
Second line protocol & & \\
Best Supportive Care (BSC) & 317 & 62.9 \\
Etoposide/Cisplatin (EP) & 2 & 0.4 \\
Gemcitabine/Cisplatin (GP) & 65 & 12.9 \\
Vinorbine/Cisplatin (NP) & 6 & 1.2 \\
Radiation (RTH) & 6 & 1.2 \\
Docetaxel (T) & 108 & 21.4 \\
Third line protocol & & \\
Best Supportive Care (BSC) & 467 & 92.7 \\
Gemcitabine/Cisplatin (GP) & 1 & 0.2 \\
Vinorbine/Cisplatin (NP) & 1 & 0.2 \\
Vinorbine (NAV) & 14 & 2.8 \\
Radiation (RTH) & 10 & 2 \\
Docetaxel (T) & 11 & 2.2 \\
First line treatment & & \\
Did not receive (n) & 73 & 14.5 \\
Received (y) & 431 & 85.5 \\
Second line treatment & & \\
Did not receive (n) & 317 & 62.9 \\
Received (y) & 187 & 37.1 \\
Third line treatment & & \\
Did not receive (n) & 467 & 92.7 \\
Received (y) & 37 & 7.3 \\
\hline
\end{tabular}

The Median PFS after first, second and third lines was 3, 4 and 2 months respectively and the median OS was 8 months. Factors which were associated with a statistically significant difference in median OS were age $<60$ years versus $\geq 60$ years (10 and 7 months respectively, $p<0.001$ ), female versus male gender (10 and 8 months respectively, $\mathrm{p}<0.001$ ), urban versus rural residence ( 9 and 8 months respectively, $\mathrm{p}=0.03$ ), smokers versus non-smokers ( 8 and 10 months respectively, $\mathrm{p}<0.001)$, patients presenting with non-neurological symptoms and those presenting with neurological symptoms (9 and 6 months respectively, $\mathrm{p}<0.001$ ) and the receiving treatment versus no treatment (10 and 5 months respectively, $\mathrm{p}<0.001)$. An interesting finding was that there was no difference in survival between the metastatic and advanced presentation groups. Cox regression analysis showed that the factors that were associated with shorter OS were age $\geq 60$ years, not receiving any line of treatment, patients presenting with neurological symptoms and male gender (see table 2 and 4).

Table 2. COX regression for the studied variables.

\begin{tabular}{|c|c|c|c|c|c|c|c|c|}
\hline \multirow{2}{*}{ Factor } & \multirow{2}{*}{ B } & \multirow{2}{*}{ SE } & \multirow{2}{*}{ Wald } & \multirow{2}{*}{ df } & \multirow{2}{*}{ Sig. } & \multirow{2}{*}{$\operatorname{Exp}(B)$} & \multicolumn{2}{|c|}{$95.0 \%$ CI for $\operatorname{Exp}(B)$} \\
\hline & & & & & & & Lower & Upper \\
\hline Age & $-.622-$ & .104 & 35.734 & 1 & .000 & .537 & .438 & .658 \\
\hline Gender & $-.913-$ & .201 & 20.523 & 1 & .000 & .401 & .271 & .596 \\
\hline Residence & .126 & .103 & 1.494 & 1 & .004 & 1.135 & 1.020 & 1.389 \\
\hline Smoking Status & .193 & .091 & 4.496 & 1 & .034 & 1.213 & 1.015 & 1.450 \\
\hline Neurological symptoms Presentation & $-.506-$ & .089 & 32.289 & 1 & .000 & .603 & .506 & .718 \\
\hline Treatment & 1.113 & .079 & 199.239 & 1 & .000 & 3.044 & 2.608 & 3.552 \\
\hline
\end{tabular}

Table 3. Factors associated with a statistically significant effect on Overall survival (OS).

\begin{tabular}{|c|c|c|c|}
\hline Factor & Median OS (in months) & 95\% Confidence Interval (in months) & p-value \\
\hline \multicolumn{4}{|l|}{ Age } \\
\hline$\geq 60$ years & 7 & $6.305-7.695$ & \multirow{2}{*}{$<0.001$} \\
\hline$<60$ years & 10 & $9.035-10.965$ & \\
\hline \multicolumn{4}{|l|}{ Gender } \\
\hline Female (F) & 10 & $8.526-11.474$ & \multirow{2}{*}{$<0.001$} \\
\hline Male (M) & 8 & $7.140-8.860$ & \\
\hline \multicolumn{4}{|l|}{ Smoking Status } \\
\hline Non-Smoker (NS) & 10 & $8.923-11.077$ & \multirow{2}{*}{$<0.001$} \\
\hline Smoker (S) & 8 & $7.199-8.801$ & \\
\hline \multicolumn{4}{|l|}{ Residence } \\
\hline Rural (R) & 8 & $7.302-8.698$ & \multirow{2}{*}{0.003} \\
\hline Urban (U) & 9 & $8.231-9.769$ & \\
\hline \multicolumn{4}{|c|}{ Neurological symptoms Presentation } \\
\hline No (n) & 9 & $8.415-9.585$ & \multirow{3}{*}{$<0.001$} \\
\hline Yes (y) & 6 & $5.433-6.567$ & \\
\hline \multicolumn{3}{|l|}{ Treatment } & \\
\hline Received Treatment & 10 & $9.429-10.571$ & \multirow{2}{*}{$<0.001$} \\
\hline No Treatment & 5 & $4.747-5.253$ & \\
\hline
\end{tabular}

\section{Discussion}

Data on clinical features and survival in NSCLC patients in Egypt are lacking ad most of it obtained through university centers. Our retrospective study analyzed 504 patients diagnosed with NSCLC who were treated at Department of Clinical Oncology and Nuclear Medicine, Ain Shams
University, Cairo-Egypt in the period from January 2008 till December 2012.

The median age of our population is younger than that reported in western population [13]. Furthermore, the median age reported from other sites in Egypt like Aswan are similar [14].

The men is predominant in our study represent $74.4 \%$ and most of our patients are smokers and in advanced stage. As 
regards the histological profile adenocarcinoma is the most common histological subtype which is similar to data reported from different population and one important reason for this is increase the accuracy of diagnosis [9].

Median overall survival and median progression free survival of patients with NSCLC were 8 months and 4 months respectively. This may be reflects that most of our patient diagnosed in advanced stage disease (74.3\%) in the study population. Furthermore, many of early stage patients could not complete the intended treatment and lead to inferior survival than expected.

On multivariate analysis we found that age $>60$, male, smoking and living in rural area as well as neurological symptoms were found to be significant adverse risk factor that affecting the survival.

Blanchon and colleagues created a prognostic index score through analytic study of 2585 non small cell lung cancer (NSCLC) patients who were treated in French general hospitals. This study revealed five independent predictors of mortality namely: age (>70 years, 1 point); sex (male, 1 point); performance status (PS) at diagnosis (reduced activity, 3 points; active $>50 \%, 5$ points; inactive $>50 \%, 8$ points; and total incapacity, 10 points); histological subtype (large-cell carcinoma, 2 points); and tumour-lymph node-metastasis (TNM) staging system (IIA or IIB, 3 points; IIIA or IIIB, 6 points; and IV, 8 points). The minimum and maximum possible point scores awarded were 0 and 22, respectively. Scores of the prognostic index were shown to have a strongly association with 4-year mortality in the development cohort [15].

Also Good performance status (PS), female sex, age greater than or equal to 70 , hemoglobin level greater than or equal to $11.0 \mathrm{~g} / \mathrm{dL}$, normal lactate dehydrogenase (LDH), normal calcium, as well as a single metastatic site were significant favorable factors for survival reported by SWOG (South-West Oncology Group) experience of advanced NSCLC [7].

From our results, we have found that there is a paradigm shift in the clinico-pathological profile of lung cancer in Egypt. Adenocarcinoma may be the commonest histological subtype in this part of the world also, provided a careful independent pathological review is done. Analysis of a larger cohort from multiple institutions is expected to reflect the true pattern. Outcome of these patients remains poor because of presentation in advanced stage and predominantly poor PS and many of them are not able to receive adequate treatment.

\section{Conclusion}

This study shows that the active treatment of patients with NSCLC continues to have an important impact on survival. The fact that rural residence could be associated with worse OS warrants further investigation. A large number of patients were not fit for further treatment after the first line which warrants more investigation as regards the available supportive measures which could elevate the performance status of the patients. The fact that EGFR mutation status testing was not available in Egypt at the time these patients received treatment, and therefore non of the patients were offered mutation directed tyrosine kinase inhibitors (TKIs), should be evaluated in further studies because EGFR testing is now available. We recommend large multicentric prospective studies comparing multimodality treatment approaches and including quality of life assessment.

\section{References}

[1] Siegel RL, Miller KD and Jemal A (2017): Cancer statistics, 2017. CA: a cancer journal for clinicians 67 (1), 7-30.

[2] Charloux A, Hedelin G, Dietemann A, Ifoundza T, Roeslin N, Pauli G, Quoix E (1997): Prognostic value of histology in patients with non-small cell lung cancer. Lung Cancer 17 (1), 123-134.

[3] Rose MC, Kostyanovskaya E, \& Huang RS. (2014): Pharmacogenomics of Cisplatin Sensitivity in Non-small Cell Lung Cancer. Genomics, Proteomics \& Bioinformatics, 12 (5), 198-209.

[4] Scagliotti, GV, De Marinis F, Rinaldi M, Crinò, L, Gridelli C, Ricci S. Italian Lung Cancer Project. (2002): Phase III Randomized Trial Comparing Three Platinum-Based Doublets in Advanced Non-Small-Cell Lung Cancer. Journal of Clinical Oncology, 20 (21), 4285-4291.

[5] Schiller JH, Harrington D, Belani C P, Langer C, Sandler A, Krook J, Eastern Cooperative Oncology Group. (2002): Comparison of Four Chemotherapy Regimens for Advanced Non-Small-Cell Lung Cancer. New England Journal of Medicine, 346 (2), 92-98.

[6] Socinski MA, Morris DE, G. A. Masters GA, and Lilenbaum R. (2003): Chemotherapeutic management of stage IV nonsmall cell lung cancer," Chest, vol. 123, supplement 1, pp. 226S-243S.

[7] Albain KS, Crowley JJ, LeBlanc M, and Livingston RB. (1991): "Survival determinants in extensive-stage non-smallcell lung cancer: the Southwest Oncology Group experience," Journal of Clinical Oncology, vol. 9, no. 9, pp. 1618-1626.

[8] Videtic GMM, Stitt LW, and Stitt LW. (2003): Continued cigarette smoking by patients receiving concurrent chemoradiotherapy for limited-stage small-cell lung cancer is associated with decreased survival. Journal of Clinical Oncology, vol. 21, no. 8, pp. 1544-1549.

[9] Abbasi S and Badheeb A. (2011): Prognostic Factors in Advanced Non-Small-Cell Lung Cancer Patients: Patient Characteristics and Type of Chemotherapy. Lung Cancer Int. 2011; 2011: 152125 .

[10] Stanley KE. (1980): Prognostic factors for survival in patients with inoperable lung cancer. Journal of the National Cancer Institute, vol. 65 , no. 1 , pp. 25-32.

[11] Finkelstein DM, Ettinger DS, and Ruckdeschel JC. (1986): Long-term survivors in metastatic non-small-cell lung cancer: an Eastern Cooperative Oncology Group study. Journal of Clinical Oncology, vol. 4, no. 5, pp. 702-709.

[12] Dela Cruz CS, Tanoue LT, \& Matthay RA (2011): Lung cancer: epidemiology, etiology, and prevention. Clinics in Chest Medicine, 32 (4), 605-644. 
[13] Cetin K, Ettinger DS, Hei YJ, O'Malley CD. (2011): Survival by histologic subtype in stage IV nonsmall cell lung cancer based on data from the Surveillance, Epidemiology and End Results Program. Clin Epidemiol. 2011; 3: 139-48.

[14] Ibrahim AS, Khaled HM, Mikhail N, Baraka H, Kamel H (2014): Cancer incidence in Egypt: results of the national population-based cancer registry program. Journal of cancer epidemiology 2014.

[15] Blanchon F, Grivaux M, Asselain B, Lebas F, Orlando J, Piquet J, Zureik M.(2006): 4-year mortality in patients with non-small-cell lung cancer: development and validation of a prognostic index. The lancet oncology 7 (10), 829-836. 